\title{
Review
}

\section{Activation of human ether-a-go-go related gene (hERG) potassium channels by small molecules}

\author{
Ping-zheng ZHOU ${ }^{1,2}$, Joseph BABCOCK${ }^{3}$, Lian-qing $\mathrm{LIU}^{2}$, Min $\mathrm{LI}^{1,3}$, Zhao-bing $\mathrm{GAO}^{1,}$ * \\ ${ }^{1}$ State Key Laboratory of Drug Research, Shanghai Institute of Materia Medica, Chinese Academy of Sciences, Shanghai 201203, \\ China; ${ }^{2}$ State Key Laboratory of Robotics, Shenyang Institute of Automation, Chinese Academy of Sciences, Shenyang 110016, China; \\ ${ }^{3}$ Department of Neuroscience, High Throughput Biology Center and Johns Hopkins Ion Channel Center, School of Medicine, Johns Hop- \\ kins University, Baltimore, Maryland 21205, USA
}

\begin{abstract}
Human ether-a-go-go related gene (hERG) potassium $\left(\mathrm{K}^{+}\right)$channels play a critical role in cardiac action potential repolarization. Mutations that reduce hERG conductance or surface expression may cause congenital long QT syndrome (LQTS). Moreover, the channels can be inhibited by structurally diverse small molecules, resulting in an acquired form of LQTS. Consequently, small molecules that increase the hERG current may be of value for treatment of LQTS. So far, nine hERG activators have been reported. The aim of this review is to discuss recent advances concerning the identification and action mechanism of hERG activators.
\end{abstract}

Keywords: Human ether-a-go-go related gene (hERG); ion channel; long QT syndrome (LQTS); activator

Acta Pharmacologica Sinica (2011) 32: 781-788; doi: 10.1038/aps.2011.70; published online 30 May 2011

\section{Discovery and structure of hERG}

In 1994, Warmke and Ganetzky first identified the human ether-a-go-go related gene (hERG) $\mathrm{K}^{+}$channel by screening a human hippocampal cDNA library with a mouse homologue of "ether-a-go-go" (EAG), a Drosophila $\mathrm{K}^{+}$channel gene ${ }^{[1]}$. Subsequently, Sanguinetti and colleagues reported similarities between the biophysical properties of the heterologously expressed hERG channel and the rapidly activating delayed rectifier $\mathrm{K}^{+}$current $\left(I_{\mathrm{Kr}}\right)$, a critical current in the phase 3 repolarization of the cardiac action potential, and confirmed that hERG encodes the a subunit of $I_{\mathrm{Kr}}{ }^{[2,3]}$. While hERG is predominately expressed in the heart, it is also found in diverse tissues including neurons, neuroendocrine glands and smooth muscle $^{[3]}$.

hERG (Kv11.1) was the 11th member of the voltage-gated $\mathrm{K}^{+}$channel family $(\mathrm{Kv})$. Like other $\mathrm{Kv}$ channels, hERG is an obligate homotetramer, with each subunit containing six transmembrane domains (S1-S6): S1-S4 compose the voltage sensor domain, while the S5, P-loop, and S6 segments form the channel pore (Figure 1). However, hERG has several features that distinguish it from other $\mathrm{Kv}$ family members. First, a conserved tyrosine found in the GYG motif of other $\mathrm{Kv}$ channel pores is replaced by a phenylalanine ${ }^{[4]}$. This aromatic

\footnotetext{
* To whom correspondence should be addressed.

E-mail zb.gao@mail.shcnc.ac.cn

Received 2011-04-01 Accepted 2011-04-25
}

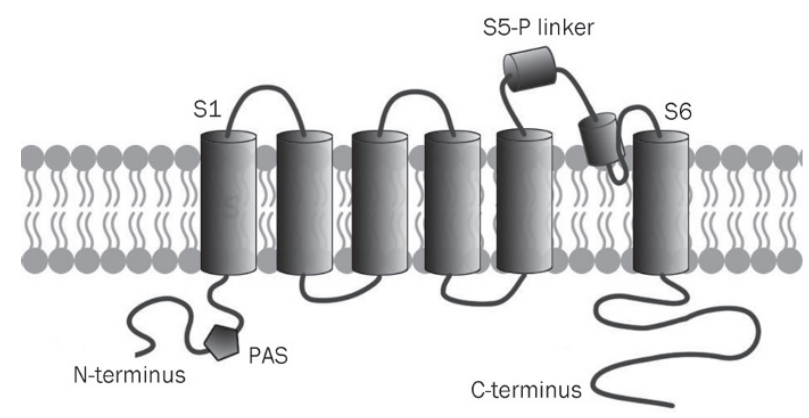

Figure 1. The basic structure of hERG channels. S1-S4 is the voltage sensor domain. S5, the P-loop, and S6 compose the pore of the channel; the termini of the protein are both intracellular.

residue, along with others lining the hERG pore, has been proposed as a determinant of promiscuous small-molecule interaction. Second, hERG's protein sequence lacks the proline$X$-proline (PXP) motif that often flanks the S6 helices of other $\mathrm{Kv}$ channels. It is generally believed that the PXP domain "kinks" this helix to reduce the volume of the pore cavity, and its absence in hERG has been suggested to play a role in promiscuous drug interactions as well. Finally, the hERG channel has a large "S5-P linker" domain between the S5 and P-loop segments that assumes an amphipathic helical arrangement in membrane mimetic sodium dodecyl sulfate (SDS) micelles and is believed to affect channel inactivation ${ }^{[5]}$. 
The intracellular termini of the hERG channel are also important for function. The N-terminus contains a Per-ArntSim (PAS) domain (about 135 amino acids), which, despite being a common motif in signaling proteins found in bacteria and plants, appears in no other mammalian ion channels. In hERG, the PAS domain modulates the deactivation of the channel following membrane depolarization. The C-terminus of the channel contains a cyclic nucleotide binding domain (CNBD), which has been linked to mutations affecting trafficking $^{[5]}$.

\section{Gating of hERG $\mathbf{K}^{+}$channels}

Although the overall structure of hERG is homologous to that of other potassium channels, its kinetic behavior is quite distinct and is characterized by comparatively slow activation and deactivation kinetics (on the order of hundreds of ms to s) but very rapid, voltage-dependent inactivation kinetics (on the order of $\mathrm{ms}$ to tens of $\mathrm{ms})^{[5,6]}$. The unusual kinetics of hERG are compatible with its function in cardiac repolarization. In the ascending phase of the action potential, as a result of slow activation and simultaneous fast inactivation, little outward current flows through hERG during depolarization. As the membrane repolarizes, hERG channels recover from inactivation much faster than they deactivate, thereby generating an outward current that peaks at about $-40 \mathrm{mV}$. This outward current through hERG is the key determinant for termination of the plateau phase of the action potential ${ }^{[5]}$ (Figure 2). Due to the importance and uniqueness of hERG gating kinetics, much of the recent work on hERG has focused on understanding the molecular rearrangements of the channel protein during the cardiac action potential.

\section{Slow activation}

As noted above, the voltage sensor of the hERG channel is the S1-S4 segment, in which the S4 helix is particularly important.
Like other Kv channels, the hERG S4 has four periodically spaced arginine residues whose positive charges, which are repelled by changes in membrane potential, are thought to drive structural rearrangement of the protein during depolarization via the S4-S5 linker ${ }^{[7]}$. Sequence alignments and hydropathy plots suggest that the overall structure of this voltage sensor domain (VSD) is homologous to that of other $\mathrm{K}^{+}$ channels. Why then are the kinetics of activation so different in hERG? To answer this question, Smith and Yellen (2002) attached a fluorescent molecule to the extracellular domain of S4 and examined the movement of this helix by fluorescence resonance energy transfer (FRET). They found that the fluorescence signal changed very slowly in response to membrane depolarization, suggesting that slow voltage sensor movement is responsible for the unusual activation gating kinetics of the channel $^{[6,8]}$. However, there still is no mechanistic explanation for the slow movement of the hERG S4 helix, though Subbiah and colleagues have identified residues K525, R528, and K538 as molecular determinants of this behavior using tryptophan scanning mutagenesis ${ }^{[9]}$.

\section{Fast voltage-dependent inactivation}

Two major mechanisms have been proposed for the inactivation of voltage-gated potassium channels ${ }^{[10]}$. The first is N-type (also called "ball and chain" type), which involves rapid occlusion of the open channel by an intracellular segment of the protein. The second is C-type, which involves a slower change in channel conformation at the extracellular mouth. There is evidence that inactivation of hERG is similar to C-type and shows voltage-dependency, even though hERG inactivation is several orders of magnitude faster than C-type inactivation in Shaker $\mathrm{K}^{+}$channels ${ }^{[3,5,11]}$. Some studies have shown that this fast inactivation limits $\mathrm{K}^{+}$efflux during depolarization, allowing hERG to function as an inward rectifier of potassium concentration $^{[11]}$.
A

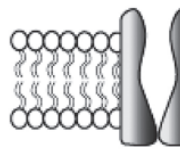

B
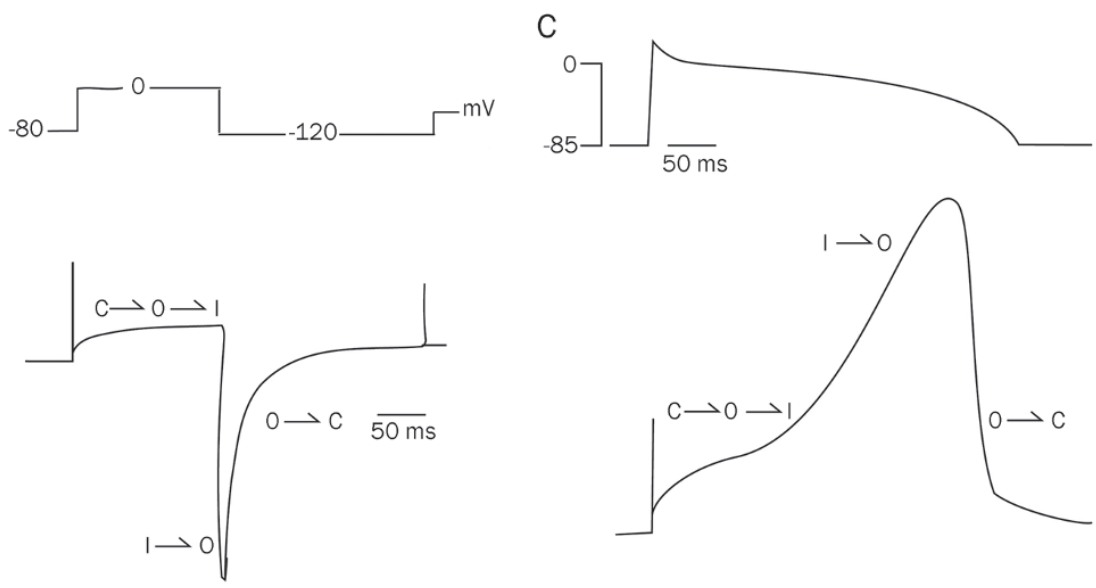

Inactivation
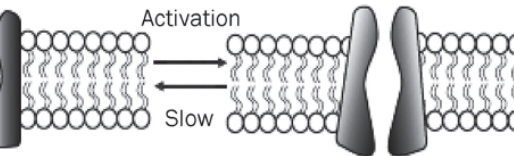

$\infty$

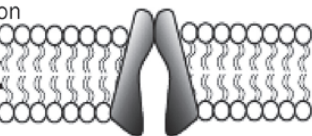


The S5-P-loop region is critical for inactivation of hERG. Many mutations in this region, most critically at $\mathbf{S 6 2 0}$ and S621, will inhibit inactivation ${ }^{[5,6]}$. S620T abolishes the inactivation of hERG, while S620A left-shifts the voltage-dependent inactivation approximately $+100 \mathrm{mV}$. Besides S620 and S621, other residues such as W585, L586, H587, L589, G590, D591, I593, and G594 have also been reported as molecular determinants of hERG channel inactivation. Furthermore, Jiang et al reported that dynamic conformational changes in the S5-P linker occur during channel gating, suggesting that this domain's mobility is critically important for hERG kinetics ${ }^{[12]}$.

It is currently still unclear why inactivation of hERG is much faster than C-type inactivation in other $\mathrm{Kv}$ channels. One explanation is that the previously mentioned tyrosine to phenylalanine substitution in the GYG pore motif may explain this isoform specific kinetics. This hypothesis comes from two experimental results: that mutation of the selectivity filter tyrosine to a phenylalanine (Y445F) in Shaker channels results in a channel with accelerated C-type inactivation and that members of the inward rectifier $\mathrm{K}^{+}$channel family, Kir6.x, contain a GFG selectivity filter and also undergo a rapid gating process that is analogous to C-type inactivation ${ }^{[6]}$.

\section{hERG and LQTS}

\section{hERG and congenital long QT syndrome}

So far, at least 13 genes have been associated with congenital LQTS ${ }^{[13]}$, including KCNQ1(LQT1), hERG(LQT2), SCN5A(LQT3), ANK2(LQT4), KCNE1(LQT5), KCNE2(LQT6), KCNJ2(LQT7), CACNA1C(LQT8), CAV3(LQT9), SCN4B(LQT10), AKAP9(LQT11), SNTA1(LQT12), and GIRK (LQT13 $^{[13,14]}$. Among these, hERG was the first reported and is the most prevalent. To date, nearly 300 different hERG mutations linked to LQT2 have been identified. Such mutations may cause loss of hERG function by one of four main effects: reduced or defective synthesis; defective trafficking from the endoplasmic reticulum (ER) to the plasma membrane (resulting in decreased surface expression); defective gating; or defective ion permeation ${ }^{[5]}$.

hERG trafficking rapidly became a focus of interest for two reasons: (1) most hERG mutations cause trafficking defects, and (2) these trafficking defective mutants can be restored by high-affinity hERG channel-blocking drugs, which then give rise to a functional $I_{\mathrm{Kr}}$ current $^{[15,16]}$. Inhibitors of hERG act as molecular chaperones to rescue transport defective mutants ${ }^{[16]}$. However, the molecular mechanism by which these small molecules function as hERG chaperones is still largely unclear, with only a single report in 2005 linking E-4031 rescue of the N470D mutant to interactions with calnexin ${ }^{[17]}$. However, the mechanism of rescue by hERG blockers is likely heterogeneous, as evidenced by the fact that molecules such as E-4031, astemizole, and cisapride are able to restore trafficking of mutants N470D and G601S but not mutations in the C-terminal such as R752W, F805C, and R823W ${ }^{[17]}$. Based on this evidence, Elizabeth $S$ Kaufman suggests that, although restoration of intracellular processing and transport is theoretically an attractive therapeutic strategy, most LQTS patients are heterozygous, and thus pharmacologic interventions that enhance the production and function of the healthy wild-type gene product may be more feasible $\mathrm{e}^{[16]}$.

\section{hERG and acquired long QT syndrome}

Unlike congenital LQTS, which may result from defects in numerous genes, almost all cases of pharmacologically induced LQTS (acquired long QT syndrome: aLQTS) have been linked to chemical blockade of the hERG channel. There is a recent paper reporting that single-nucleotide polymorphisms in other proteins that interact with hERG may be inhibited by small molecules, and thus also cause a LQTS ${ }^{[18]}$. Indeed, it is thought that hERG is a promiscuous target that binds structurally diverse small molecules.

Until now, aLQTS has resulted in many drugs being removed from the market or terminated during clinical development. Examples include several non-cardiac drugs that have been withdrawn or given strict limitation for use, including terfenadine, lidoflazine, astemizole, sertindole, levomethadyl, droperidol, cisapride and grepafloxacin (Table $1)^{[19]}$. Thus, potential blockade of cardiac $I_{\mathrm{Kr}}$ becomes a necessary pre-clinical assessment for candidate drugs. Structural determinants for this chemical blockade include a tyrosine at position 652 and a phenylalanine at $656^{[5]}$. Alanine scanning mutagenesis has demonstrated that these two residues dictate the high-affinity binding of many drugs that inhibit hERG. However, the docking mode of different drugs is not the same, and computational modeling suggests that many drugs may utilize diverse binding conformations, coordinating multiple residues both within and between the subunits of the channel. Thus, the complex interactions between drugs and hERG complicate in silico predictions of the hERG inhibition by novel therapeutic compounds.

\section{hERG activators}

As mentioned above, hERG mutations cause congenital LQTS and a wide variety of drugs of different classes and structures bind to hERG, leading to aLQTS. However, hERG defects have also been linked to other diseases such as stressmediated arrhythmias, diabetes and myocardial ischemia induced arrhythmias ${ }^{[20]}$. Physiologically potentiating hERG would accelerate action potential repolarization and shorten the duration of the action potential. hERG channel activators can enhance channel function by accelerating myocardial repolarization, an effect that has been demonstrated by animal experiments, and they are considered potential therapeutics for LQTS. Indeed, hERG activators may become a novel class of antiarrhythmics, as reports have suggested that such compounds can reduce electrical heterogeneity in the myocardium and thereby the possibility of re-entry ${ }^{[20,21]}$. However, due to the limitations of existing high throughput screening methods and difficulties in assaying the channel on a large scale, few activators have been reported. Most of the existing activators originated from combinatorial chemistry libraries, includ-

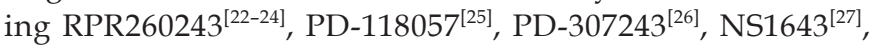
NS3623 ${ }^{[28]}$, A-935142 $2^{[29]}$, ICA-105574 $4^{[30]}$, and KB130015 ${ }^{[31]}$. Addi- 
Table 1. Summary of nine non-cardiac drugs withdrawn for hERG toxicity.

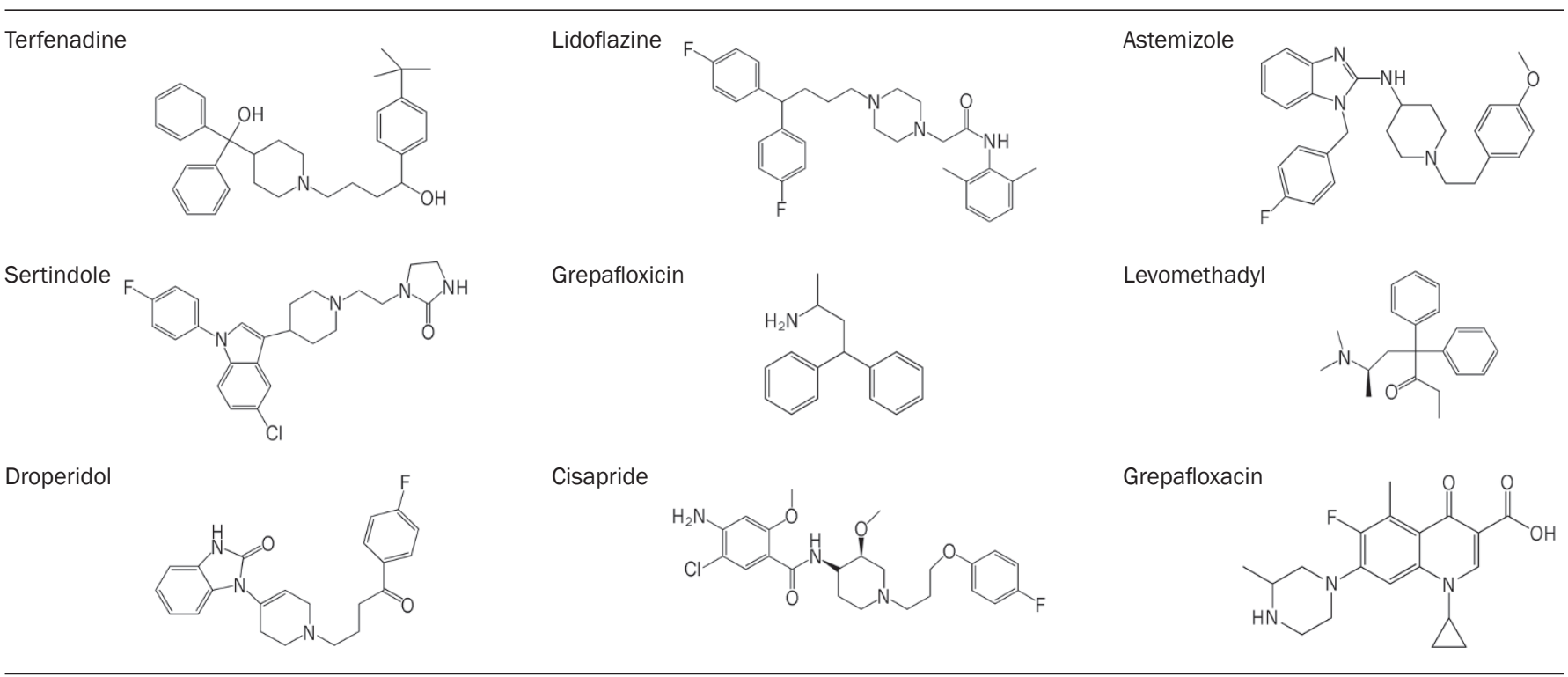

tionally, a natural product, mallotoxin, has also been shown to activate $\mathrm{hERG}^{[32]}$. Below, we review the literature on individual compounds.

\section{RPR260243}

RPR260243 [(3R,4R)-4-[3-(6-methoxy-quinolin-4-yl)-3-oxopropyl]-1-[3-(2,3,5-trifluoro-phenyl)-prop-2-yn-1-yl]-piperidine-3-carboxylic acid] was the first reported hERG channel activator. RPR260243 dramatically slows current deactivation in patch-clamp experiments, and its effect is temperature and voltage dependent. Though it is a weak inhibitor of the L-type $\mathrm{Ca}^{2+}$ channel, RPR260243 has no significant effects on the human cardiac $\mathrm{Na}^{+}$channel or the KCNQ1/KCNE1 cardiac $\mathrm{K}^{+}$channel, which are also linked with LQTS, thus showing high selectivity for hERG ${ }^{[22]}$. Interestingly, RPR260243 inhibits the erg3 channel, which is in the same family as hERG, and a single 55 residue may account for this difference in pharmacology (Thr556 in hERG, Ile558 in rERG3). A Thr in this position favors agonist activity, whereas an Ile reveals a secondary blocking effect of RPR260243 ${ }^{[23]}$. Additionally, RPR260243 enhances the delayed rectifier current in guinea pig myocytes and can, to some extent, reverse dofetilide-induced prolongation of action potential. Physiologically, it has been reported that RPR260243 can increase the T-wave amplitude, prolong the PR interval and shorten the QT interval in guinea pig hearts $^{[22]}$.

\section{PD-118057}

PD-118057 [2-(4-[2-(3,4-dichloro-phenyl)-ethyl]-phenylamino)benzoic acid] was first reported by Zhou and colleagues ${ }^{[25]}$. It primarily enhances the peak amplitude of the hERG tail current in a dose-dependent manner. PD-118057 shows no major effect on $I_{\mathrm{Na}}, I_{\mathrm{Ca}}, I_{\mathrm{K} 1}$, or $I_{\mathrm{Ks}}$, and it shortens the action potential duration and QT interval in arterially perfused rabbit ven- tricular wedge preparations and prevents QT prolongation by dofetilide. Mechanistically, Zhou et al reported that the compound did not affect the voltage dependence or kinetics of gating, nor did its activity require the open conformation of the channel. Later results by Sanguinetti and colleagues suggest that PD-118057 activates the hERG channel mainly by attenuating inactivation ${ }^{[33]}$. They also found that $10 \mu \mathrm{mol} / \mathrm{L}$ PD-118057 shifted the half-point of hERG channel inactivation by $+19 \mathrm{mV}$, increased peak outward current amplitude by $136 \%$, and enhanced $\mathrm{K}^{+}$conductance.

\section{PD-307243}

PD-307243 [2-[2-(3,4-dichloro-phenyl)-2,3-dihydro-1Hisoindolin-5-ylamino]-nicotinic acid] significantly enhances hERG currents by slowing channel deactivation and inactivation. At potentials from -120 to $-40 \mathrm{mV}$, PD-307243 induces instantaneous hERG current with little decay. When the membrane potential is higher than $-40 \mathrm{mV}$, PD-307243 induces an $I_{\text {to }}$-like upstroke of hERG current. This $I_{\text {to }}$-like current may result from slowed channel inactivation and deactivation, and this effect can be only observed once the channel is in the open state, which may also explain the compound's use dependence. Additionally, hERG reversal potential was not altered in the presence of $3 \mu \mathrm{mol} / \mathrm{L}$ PD-307243, suggesting that the compound does not affect the selectivity filter of the channel ${ }^{[26]}$.

\section{NS1643}

Casis and colleagues (2006) reported that NS1643 [1,3-bis(2-hydroxy-5-trifluoromethyl-phenyl)-urea] activates hERG channels expressed in Xenopous oocytes in a concentrationand voltage-dependent manner ${ }^{[27]}$. At a depolarization voltage of $-10 \mathrm{mV}$, the compound's $\mathrm{EC}_{50}$ value is $10.4 \mu \mathrm{mol} / \mathrm{L}$. While NS1643 strongly affects channel inactivation by right 
shifting the voltage-inactivation curve by $+21 \mathrm{mV}$ at $10 \mu \mathrm{mol} /$ $\mathrm{L}$ and $+35 \mathrm{mV}$ at $30 \mu \mathrm{mol} / \mathrm{L}$, it has no effect on the activation of the channel. In the absence of inactivation, NS1643 does not enhance outward current magnitude ${ }^{[27]}$. However, $\mathrm{Xu}$ et al (2008) reported that NS1643 can also left-shift the voltage-dependent activation curve ${ }^{[34]}$. In guinea pig cardiac myocytes, $10 \mu \mathrm{mol} / \mathrm{L}$ NS1643 can activate $I_{\mathrm{Kr}}$ and significantly shorten the action potential duration ${ }^{[27]}$.

\section{NS3623}

NS3623 [N-(4-bromo-2-(1H-tetrazol-5-yl)phenyl)-N'-(3-trifluoromethyl-phenyl)urea], originally identified as a chloride channel blocker ${ }^{[35]}$, activates hERG channels expressed in Xenopous oocytes, with an $\mathrm{EC}_{50}$ value of $79.4 \mu \mathrm{mol} / \mathrm{L}^{[28]}$. NS3623 mainly affects the voltage dependence of channel inactivation by right-shifting the half point of inactivation by $+17.7 \mathrm{mV}$. NS3623 also slows channel inactivation. Similar to results found using NS1643, inactivation defective mutants S620T and S631A are not sensitive to NS3623, supporting the conclusion that these two compounds have similar mechanisms of action on $\mathrm{hERG}^{[28]}$.

\section{A-935142}

A-935142 [\{4-[4-(5-trifluoromethyl-1H-pyrazol-3-yl)-phenyl]cyclohexyl\}-acetic acid)] was reported in 2009 as a hERG channel activator that enhances the amplitude of step and tail current in a concentration- and voltage-dependent manner. Current-voltage curves in the presence of $60 \mu \mathrm{mol} / \mathrm{L}$ A-935142 suggest that the compound enhances both the outward and the inward $\mathrm{K}^{+}$currents. Unlike previously reported activators, A-935142 simultaneously affects channel activation, deactivation and inactivation. Specifically, $60 \mu \mathrm{mol} / \mathrm{L}$ of A-935142 significantly accelerates the activation time constant of hERG channels from $164 \pm 24 \mathrm{~ms}$ to $100 \pm 17 \mathrm{~ms}$ and left-shifts the voltage-dependence of activation. A-935142 also reduces the rate of inactivation, right-shifts the voltage-dependence of inactivation, and slows hERG channel deactivation at voltage potentials from -120 to $-70 \mathrm{mV}^{[29]}$.

\section{ICA-105574}

Gerlach and colleagues recently described a compound, ICA105574 [3-nitro-N-(4-phenoxyphenyl) benzamide], that activates hERG channels, mainly by affecting channel inactivation, with a magnitude much larger than that of previously reported activators. Two micromolar ICA-105574 shifts the mid-point of the voltage-dependent inactivation by $>180 \mathrm{mV}$ from $-86 \mathrm{mV}$ to $+96 \mathrm{mV}$. Consistent with this observation, 2 $\mu \mathrm{mol} / \mathrm{L}$ ICA-105574 potentiates outward current amplitude ten fold, with an $\mathrm{EC}_{50}$ of $0.5 \pm 0.1 \mu \mathrm{mol} / \mathrm{L}$. In addition to effects on channel inactivation, high concentrations of the compound ( $3 \mu \mathrm{mol} / \mathrm{L})$ can also left-shift the voltage dependence of channel activation by $-11 \mathrm{mV}$ and slow channel deactivation 2-fold. Finally, ICA-105574 induces a concentration-dependent shortening of action potential duration $(>70 \%$ at $3 \mu \mathrm{mol} / \mathrm{L})$ in isolated guinea pig ventricular cardiac myocytes. This effect can be prevented by hERG inhibitor E-4031, supporting the conclusion that ICA-105574 increases hERG channel function through direct action on the protein ${ }^{[30]}$.

\section{KB130015}

KB130015 [(2-methyl-3-(3,5-diiodo-4-carboxymethoxybenzyl) benzofuran)] is a derivative of amiodarone, a potent hERG blocker ${ }^{[36]}$. Because of the similarity, Gessner et al assumed KB130015 inhibited hERG ${ }^{[31]}$. Unexpectedly, they found that, while KB130015 does inhibit native and recombinant hERG at high voltages, it can activate both forms of the channel at low voltages. KB130015 accelerates activation by 4-fold and left -shifts the voltage-dependent activation curve by $-16 \mathrm{mV}$, with an $\mathrm{EC}_{50}$ value of $12 \mu \mathrm{mol} / \mathrm{L}$. Based on its similarity to amiodarone, KB130015 presumably binds to the hERG pore from the cytosolic side and functionally competes with blockade by amiodarone and other canonical inhibitors at this site ${ }^{[31]}$.

\section{Mallotoxin}

Mallotoxin (MTX) [1-(6-(3-acetyl-2,4,6-trihydroxy-5-methylbenzyl)-7-hydroxy-2,2-dimethyl-2H-chromen-8-yl)-3-phenylprop-2-en-1-one], an extract of the tree Mallotus phillippinensis, is the only natural product reported to activate hERG. It was previously shown to inhibit protein kinase $\mathrm{C}$ (PKC), $\mathrm{Ca}^{2+}$ / calmodulin-dependent protein kinase II and III, and elongation factor-2 kinase. Zeng et al (2006) discovered that MTX can enhance both step and tail hERG current, with EC $_{50}$ values of 0.34 and $0.52 \mu \mathrm{mol} / \mathrm{L}$, respectively ${ }^{[32]}$. The potency of MTX is at least ten fold higher than previously reported hERG activators, including PD-118057, NS1643, and RPR260243. Furthermore, the mechanism by which MTX potentiates hERG is unique in comparison to these synthetic molecules. It mainly affects channel activation (left-shifting the activation curve by $+24 \mathrm{mV}$ at $2.5 \mu \mathrm{mol} / \mathrm{L} \mathrm{MTX)}$ and deactivation without modulating inactivation. Using pre-recorded cardiac action potentials, $2.5 \mu \mathrm{mol} / \mathrm{L} \mathrm{MTX} \mathrm{increases} \mathrm{the} \mathrm{total} \mathrm{number} \mathrm{of} \mathrm{potassium}$ ions passed through hERG channels by $\sim 5$-fold $^{[32]}$.

\section{Binding site of small-molecule hERG activators}

Understanding the binding site of agonists is helpful for investigating channel conformation and gating, particularly for rational drug design. However, the exact molecular determinants of hERG activator function remain unresolved, with most of the knowledge about possible binding sites derived from mutagenesis experiments.

Sangunetii et al found that two groups of residues have different effects on RPR260243 ${ }^{[24,37]}$. One group, including L553, F557 (S5), and N658, V659 (S6), affects the inactivation and deactivation effect of RPR260243 on the hERG channel, while mutations in the other group of residues, including V549, L550 (S4-S5 linker), and I662, L666, Y667 (intracellular S6 segment), only hinder the transition to the closed state of the channel. In another study by Sangunetii et al about possible binding sites of PD-118057 ${ }^{[33]}$, they focused on the S5-P-S6 domain. Through alanine scanning mutagenesis of this region, four mutants (F619A, L622C, I639A, and L646A) were identified that display the lowest agonist activity in all tested mutants. Molecular 
simulations suggested that PD-118057 interacted with F619 in the pore domain of one subunit and L646 (S6) of an adjacent subunit, which together form a hydrophobic binding pocket, reducing channel inactivation and increasing channel open possibility.

Limited binding site information about PD-307243 and NS1643 was reported by $\mathrm{Xu}$ and Tseng in 2008 ${ }^{[34]}$. Because both compounds act on the extracellular side of the channel and significantly slow channel inactivation, the authors speculated that these compounds may act on the pore domain. Perturbation of the conformation of the outer vestibule/external pore entrance (by cysteine substitution at high-impact positions or cysteine side chain modification at intermediateimpact positions) prevented the activation effect of NS1643 but not that of PD-307243, suggesting that NS1643 may bind to this domain. Further pharmacological experiments showed that the effects of PD-307243 could be abolished by TPeA ${ }^{+}$and dofetilide (both hERG inhibitors that block the pore), which supports the conclusion that PD-307243 may be a "pore-modifier" (Table 2) ${ }^{[34]}$.

\section{Outlook and challenges}

LQTS is responsible for many sudden deaths before age 20 . Current treatments for LQTS include beta-adrenergic receptor blockers, left cardiac sympathetic denervation or, in the worst cases, implantation of cardiac defibrillators ${ }^{[24,38]}$. However, pharmacologic treatment is not always effective, and both surgery and implanted devices are expensive and require invasive procedures ${ }^{[24,39]}$. Acute episodes of drug-induced LQTS are treated with magnesium sulfate administration and discontinued use of the suspect medication. Activation of hERG could provide an alternative and more specific treatment for acquired or congenital LQTS. In addition, hERG activators may become a novel class of antiarrhythmics because, as mentioned above, they can reduce electrical heterogeneity in the myocardium and, thereby, the possibility of re-entry ${ }^{[20,21]}$ However, this idea has yet to be confirmed clinically. A recent report in PubChem described a co-drug screen aiming to protect against the development of aLQTS by a high-throughput method (AID: 1680). Compounds that exhibit such activity would be expected to include neutral antagonists that bind to the same site as hERG blockers but do not favor the "blocked conformation" of the pore. Alternatively, these compounds may be hERG agonists that competitively bind to the same site as hERG inhibitors or compounds that prevent the access of a hERG inhibitor to its cognate binding site at the intracellular aspect of the pore region. Although it remains to be verified whether these compounds would be effective, this strategy does provide a new idea for the treatment of aLQTS.

The structural and mechanistic heterogeneity of the currently reported hERG activators suggests that hERG channels may have different agonist binding sites, a phenomenon that has been observed in voltage-gated KCNQ channel activators. However, so far, studies on the binding site by hERG agonists are still descriptive, mostly relying on inference from mutagenesis data for RPR260243 and PD118057. However, activation of hERG by small molecules may be a double-edged sword from a clinical perspective because excessive potentiation may chemically induce short QT syndrome ${ }^{[40]}$. Moreover, there are no reports from animal models or human clinical data about drug-induced short QT syndrome, indicating that the safety of hERG agonists remains to be tested.

\section{Acknowledgements}

This work was supported by National Natural Science Foundation of China grant 81072579 and open funds of State Key Laboratory of Robotics RLO201013.

We thank Dr Guo-yuan HU for valuable discussions and comments on the manuscript.

\section{References}

1 Warmke JW, Ganetzky B. A family of potassium channel genes related to eag in Drosophila and mammals. Proc Natl Acad Sci U S A 1994; 91: 3438-42.

2 Sanguinetti MC, Jiang C, Curran ME, Keating MT. A mechanistic link between an inherited and an acquired cardiac arrhythmia: HERG encodes the $I_{\mathrm{Kr}}$ potassium channel. Cell 1995; 81: 299-307.

3 Shen XZ, Wu J, Lin JJ. HERG and arrhythmia. Adv Cardiovasc Dis 2008; 29: 436-40.

4 Lagrutta AA, Trepakova ES, Salata JJ. The hERG channel and risk of drug-acquired cardiac arrhythmia: an overview. Curr Top Med Chem 2008; 8: 1102-12.

5 Perrin MJ, Subbiah RN, Vandenberg JI, Hill AP. Human ether-a-gogo related gene (hERG) $\mathrm{K}^{+}$channels: function and dysfunction. Prog Biophys Mol Biol 2008; 98: 137-48.

6 Vandenberg JI, Torres AM, Campbell TJ, Kuchel PW. The HERG K ${ }^{+}$ channel: progress in understanding the molecular basis of its unusual gating kinetics. Eur Biophys J 2004; 33: 89-97.

7 Piper DR, Sanguinetti MC, Tristani-Firouzi M. Voltage sensor movement in the hERG $\mathrm{K}^{+}$channel. Novartis Found Symp 2005; 266: 46-52.

8 Smith PL, Yellen G. Fast and slow voltage sensor movements in HERG potassium channels. J Gen Physiol 2002; 119: 275-93.

9 Subbiah RN, Kondo M, Campbell TJ, Vandenberg Jl. Tryptophan scanning mutagenesis of the HERG $\mathrm{K}^{+}$channel: the $\mathrm{S} 4$ domain is loosely packed and likely to be lipid exposed. J Physiol 2005; 569: 367-79.

10 Baukrowitz T, Yellen G. Modulation of $\mathrm{K}^{+}$current by frequency and external $\left[\mathrm{K}^{+}\right]$: a tale of two inactivation mechanisms. Neuron 1995; 15: 951-60.

11 Smith PL, Baukrowitz T, Yellen G. The inward rectification mechanism of the HERG cardiac potassium channel. Nature 1996; 379: 833-6.

12 Jiang M, Zhang M, Maslennikov IV, Liu J, Wu DM, Korolkova YV, et al. Dynamic conformational changes of extracellular S5-P linkers in the hERG channel. J Physiol 2005; 569: 75-89.

13 Hedley PL, Jørgensen P, Schlamowitz S, Wangari R, Moolman-Smook J, Brink PA, et al. The genetic basis of long QT and short QT syndromes: a mutation update. Hum Mutat 2009; 30: 1486-511.

14 Roden DM, Lazzara R, Rosen M, Schwartz PJ, Towbin J, Vincent GM. Multiple mechanisms in the long-QT syndrome. Current knowledge, gaps, and future directions. The SADS Foundation Task Force on LQTS. Circulation 1996; 94: 1996-2012.

15 Charpentier F, Merot J, Loussouarn G, Baro I. Delayed rectifier $\mathrm{K}^{+}$ currents and cardiac repolarization. J Mol Cell Cardiol 2010; 48: $37-44$. 
Table 2. Summary of 8 HERG $\mathrm{K}^{+}$channels activators.

Compound name Structure

PD-118057<smiles>O=C(O)c1ccccc1Nc1ccc(CCc2ccc(Cl)c(Cl)c2)cc1</smiles>

PD-307243<smiles>O=Cc1cccnc1Nc1ccc2c(c1)CN(c1ccc(Cl)c(Cl)c1)C2</smiles>

NS1643<smiles>Cc1ccc(O)c(NC(=O)Nc2cc(C(F)(F)F)ccc2O)c1</smiles>

NS3623<smiles>O=C(Nc1cccc(C(F)(F)F)c1)Nc1ccc(Br)cc1-c1nnn[nH]1</smiles>

A-935142<smiles>O=C(O)CC1CCC(c2ccc(-c3cc(C(F)(F)F)[nH]n3)cc2)CC1</smiles>

ICA-10557<smiles>O=C(Nc1ccc(Oc2ccccc2)cc1)c1cccc([N+](=O)[O-])c1</smiles>

KB130015<smiles>Cc1cc(Cc2c(C)oc3ccccc23)cc(I)c1OCC(=O)O</smiles>

Mallotoxin (MTX)

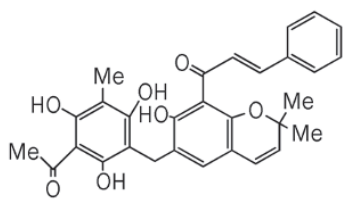

Mechanism of action

Slows deactivation

Attenuates inactivation

Slows channel deactivation and inactivation

Attenuates inactivation

Attenuates inactivation

No report

No report

Accelerates activation

Attenuates inactivation

and slow deactivation

Mainly attenuates or remove channel inactivation, besides at high concentration it also accelerates activation and slows deactivation

Accelerates activation

Left-shifts the activation curve, and slows deactivation process ductance channels hERG

No report

No report
Interacts with residues in the S4-S5 linker or cytoplasmic ends of the S5 and S6 domains

Contacts the pore helix of hERG channels to attenuates inactivation and enhances $\mathrm{K}^{+}$con-

May works as a "poremodifier" of the hERG

Binds to the outer vestibule/pore entrance of

No report

No effect on $I_{\mathrm{Na}}, I_{\mathrm{Ca}, \mathrm{L}}, I_{\mathrm{K} 1}$, and $I_{\mathrm{ks}}$

Activates $I_{\mathrm{ks}}$ and $I_{\mathrm{Ca}, \mathrm{L}}$;

no effect on $I_{\text {to }}$ and Nav1.5

No effect on human cardiac

[22-24]

-type $\mathrm{Ca}^{2+}$ channels, inhibits erg3 channel

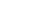

No effect on $I_{\mathrm{Ks}}, I_{\text {Kur }}, \mathrm{Kv} 4.3, \quad$ [28] $I_{\mathrm{Ca}, \mathrm{T}}, I_{\mathrm{Na}}$, activates $I_{\mathrm{Ca}, \mathrm{L}}$

No report

No report

Presumably binds to No report pore domain from the cytosolic-side

No report
16 Kaufman ES, Ficker E. Is restoration of intracellular trafficking clinically feasible in the long QT syndrome? The example of HERG mutations. J Cardiovasc Electrophysiol 2003; 14: 320-2.

17 Gong Q, Jones MA, Zhou, Z. Mechanisms of pharmacological rescue of trafficking-defective hERG mutant channels in human long QT syndrome. J Biol Chem 2006; 281: 4069-74.

18 Berger SI, Ma'ayan A, Iyengar R. Systems pharmacology of arrhythmias. Sci Signal 2010; 3: ra30. 
19 Roden DM. Drug-induced prolongation of the QT interval. N Engl J Med 2004; 350: 1013-22.

20 Guan FY, Yang SJ. HERG K ${ }^{+}$channel, the target of anti-arrhythmias drugs. Yao Xue Xue Bao 2007; 42: 687-91.

21 Wulff $\mathrm{H}$, Castle NA, Pardo LA. Voltage-gated potassium channels as therapeutic targets. Nat Rev Drug Discov 2009; 8: 982-1001.

22 Kang J, Chen XL, Wang H, Ji J, Cheng H, Incardona J, et al. Discovery of a small molecule activator of the human ether-a-go-go-related gene (HERG) cardiac $\mathrm{K}^{+}$channel. Mol Pharmacol 2005; 67: 827-36.

23 Perry M, Sanguinetti MC. A single amino acid difference between ether-a-go-go-related gene channel subtypes determines differential sensitivity to a small molecule activator. Mol Pharmacol 2008; 73 : 1044-51.

24 Perry M, Sachse FB, Sanguinetti MC. Structural basis of action for a human ether-a-go-go-related gene 1 potassium channel activator. Proc Natl Acad Sci U S A 2007; 104: 13827-32.

25 Zhou J, Augelli-Szafran CE, Bradley JA, Chen X, Koci BJ, Volberg WA, et al. Novel potent human ether-a-go-go-related gene ( $\mathrm{hERG}$ ) potassium channel enhancers and their in vitro antiarrhythmic activity. Mol Pharmacol 2005; 68: 876-84.

26 Gordon E, Lozinskaya IM, Lin Z, Semus SF, Blaney FE, Willette RN, et al. 2-[2-(3,4-dichloro-phenyl)-2,3-dihydro-1H-isoindol-5-ylamino]nicotinic acid (PD-307243) causes instantaneous current through human ether-a-go-go-related gene potassium channels. Mol Pharmacol 2008; 73: 639-51.

27 Casis O, Olesen SP, Sanguinetti MC. Mechanism of action of a novel human ether-a-go-go-related gene channel activator. Mol Pharmacol 2006; 69: 658-65.

28 Hansen RS, Diness TG, Christ T, Wettwer E, Ravens U, Olesen SP, et al. Biophysical characterization of the new human ether-a-go-gorelated gene channel opener NS3623 [N-(4-bromo-2-(1H-tetrazol-5yl)-phenyl)- $N$ '-(3'-trifluoromethylphenyl)urea]. Mol Pharmacol 2006; 70: 1319-29.

29 Su Z, Limberis J, Souers A, Kym P, Mikhail A, Houseman K, et al. Electrophysiologic characterization of a novel hERG channel activator. Biochem Pharmacol 2009; 77: 1383-90.

30 Gerlach AC, Stoehr SJ, Castle NA. Pharmacological removal of human ether-a-go-go-related gene potassium channel inactivation by 3-nitro$\mathrm{N}$-(4-phenoxyphenyl) benzamide (ICA-105574). Mol Pharmacol 2010; 77: 58-68.

31 Gessner G, Macianskiene R, Starkus JG, Schonherr R, Heinemann SH. The amiodarone derivative KB130015 activates hERG1 potassium channels via a novel mechanism. Eur J Pharmacol 2010; 632: 52-9.

32 Zeng H, Lozinskaya IM, Lin Z, Willette RN, Brooks DP, Xu X, et al. Mallotoxin is a novel human ether-a-go-go-related gene ( $h E R G$ ) potassium channel activator. J Pharmacol Exp Ther 2006; 319: 957-62.

33 Perry M, Sachse FB, Abbruzzese J, Sanguinetti MC. PD-118057 contacts the pore helix of hERG1 channels to attenuate inactivation and enhance $\mathrm{K}^{+}$conductance. Proc Natl Acad Sci U S A 2009; 106 : 20075-80.

$34 \mathrm{Xu} \mathrm{X}$, Recanatini M, Roberti M, Tseng GN. Probing the binding sites and mechanisms of action of two human ether-a-go-go-related gene channel activators, 1,3-bis-(2-hydroxy-5-trifluoromethyl-phenyl)-urea (NS1643) and 2-[2-(3,4-dichloro-phenyl)-2,3-dihydro-1H-isoindol5-ylamino]-nicotinic acid (PD307243). Mol Pharmacol 2008; 73 : 1709-21.

35 Bennekou P, de Franceschi L, Pedersen O, Lian L, Asakura T, Evans G, et al. Treatment with NS3623, a novel Cl-conductance blocker, ameliorates erythrocyte dehydration in transgenic SAD mice: a possible new therapeutic approach for sickle cell disease. Blood 2001; 97: 1451-7.

36 Ridley JM, Milnes JT, Witchel HJ, Hancox JC. High affinity HERG $\mathrm{K}^{+}$channel blockade by the antiarrhythmic agent dronedarone: resistance to mutations of the S6 residues Y652 and F656. Biochem Biophys Res Commun 2004; 325: 883-91.

37 Perry M, Sanguinetti M, Mitcheson J. Revealing the structural basis of action of hERG potassium channel activators and blockers. J Physiol 2010; 588: 3157-67.

38 Schwartz PJ. The congenital long QT syndromes from genotype to phenotype: clinical implications. J Intern Med 2006; 259: 39-47.

39 Gow RM. Effectiveness and limitations of beta-blocker therapy in congenital long-QT syndrome. Circulation 2001; 103: E24.

40 Bjerregaard P, Jahangir A, Gussak I. Targeted therapy for short QT syndrome. Expert Opin Ther Targets 2006; 10: 393-400. 